\title{
Estudo do Comportamento Mecânico, Termomecânico e Morfológico de Misturas de Poliestireno/Composto de Borracha Reciclada (SBR)
}

\section{Study of Mechanical, Thermomechanical and Morphological Behavior of the Polystyrene/ Recycled Rubber (SBR) Blend}

\author{
Carlos Bruno Barreto Luna , Divânia Ferreira da Silva , \\ Edcleide Maria Araújo ${ }^{1}$, Tomás Jeferson Alves de Melo ${ }^{1}$, \\ Amanda Dantas de Oliveira ${ }^{1}$
}

\author{
${ }^{1}$ Unidade Acadêmica de Engenharia de Materiais, UAEMa/CCT/UFCG, Rua: Aprígio Veloso, 882, cep 58429-140, \\ Campina Grande, PB. e-mail: brunobarretodemaufcg@hotmail.com; divaniaf@yahoo.com.br; \\ edcleide@dema.ufcg.edu.br; tomas@dema.ufcg.edu.br. \\ ${ }^{2}$ Departamento de Engenharia de Materiais, DEMA/UFSCAR, Rua: Washington Luis, 235, cep 13565-905, São Carlos, \\ SP. e-mail: amandaoliveira82@gmail.com
}

\begin{abstract}
RESUMO
Misturas de polímeros constituem alternativa para a obtenção de materiais com propriedades que, em geral, não são encontradas em um único material. O objetivo deste trabalho foi produzir misturas poliméricas a partir de uma matriz de poliestireno (PS) com composto de borracha reciclada (SBR) visando à obtenção de propriedades superiores ao PS. As misturas de poliestireno/composto de borracha reciclada foram preparadas em uma extrusora de rosca dupla corrotacional, com adição de agente compatibilizante e, posteriormente, os grânulos extrusados foram moldados por injeção. Estas foram caracterizadas por ensaio mecânico de tração, análise térmica-dinâmico-mecânica (DMTA), temperatura de deflexão térmica (HDT) e microscopia eletrônica de varredura (MEV). Observou-se que não houve perdas significativas da HDT e da resistência à tração. O ensaio de DMTA evidencia a presença de dois picos de transição distintos, um referente a Tg do PS e o outro referente a Tg do polibutadieno (PB). A morfologia ilustrou uma redução da quantidade de vazios e do tamanho das partículas quando a mistura foi compatibilizada com 5\% de SBS. As misturas de PS/SBR são uma alternativa para o reaproveitamento dos resíduos de calçados, bem como, uma forma de agregar valor a um material que seria descartado.
\end{abstract}

Palavras-chave: Misturas poliméricas, resíduos de borracha, compatibilizante, propriedades.

\section{ABSTRACT}

The combination of polymer is an alternative to obtain materials with properties that, in general, are not found in a single material. The goal of this work was to produce polymer blend with polystyrene (PS) as a matrix and recycled rubber compound (SBR) as a minor phase, in order to obtain superior properties to PS. The combination of polystyrene/ composed recycled rubber was prepared in a double screw corrotational extruder and, subsequently, the extruded pellets were molded by injection. These were characterized by mechanical tests, dynamic mechanical thermal analysis (DMTA), heat deflection temperature (HDT) and scanning electron microscopy (SEM). It was observed that there was no significant loss in HDT and on tensile strength. The DMTA tests showed the presence of two peaks transition, a reference to the PS Tg and another regarding the Tg of PB. The morphology showed a reduction in the quantity of voids and in the size of the particles when the mixture was compatibilized with 5\% SBS. The PS/SBR blends are an alternative to the reuse of footwear wastes, as well as to add value to a material that would be discarded. 
Keywords: polymer blends, rubber wastes, compatibilizer, properties.

\section{INTRODUÇÃO}

O desenvolvimento de processos para modificação de mistura de polímeros é de fundamental importância para combinar as propriedades de seus componentes em um só produto. Como por exemplo, as misturas poliméricas, que são obtidas a partir da mistura física de dois ou mais polímeros, visando à obtenção de novos materiais com propriedades desejadas [1-2]. As misturas possuem vantagens como a facilidade de ajustar as propriedades dos polímeros às necessidades de uso, bem como, gerar propriedades mecânicas, químicas ou de barreiras desejadas e com baixo custo, quando comparado à síntese de novos polímeros [3] .

No desenvolvimento de misturas a escolha adequada dos componentes pode proporcionar uma redução de custo do composto final e/ou do produto a ser moldado com esse material [4]. A possibilidade de alterar as propriedades de um polímero por meio da simples adição de outros polímeros com custos menores é mais vantajoso do que alterar o processo de polimerização ou sintetizar um novo polímero. Portanto, as misturas de polímeros é uma estratégia bastante atrativa para a indústria [믐].

O poliestireno (PS) é um dos termoplásticos mais utilizados pela indústria de transformação de polímeros. A produção comercial do PS iniciou em 1930, na Alemanha, e este polímero caracteriza-se pela sua transparência, facilidade de processamento, estabilidade térmica, baixa densidade, alto módulo de elasticidade, excelentes propriedades elétricas e baixo custo [7]. Entretanto, o PS possui limitações em algumas propriedades mecânicas como ductilidade e resistência [8]. Para contornar este problema, a principal técnica utilizada pelas indústrias de polímeros tem sido o seu reforço com partículas de borrachas [9-10]. O reforço com elastômeros é a técnica mais utilizada por grande parte das indústrias de polímeros para aumentar a resistência à fratura e a tenacidade desses materiais [11]. Nas misturas de termoplásticos com elastômeros, quando se submete o material a uma carga, as partículas elastoméricas dispersas podem concentrar ou absorver tensões, provocando uma alteração do estado de tensão da fase matricial e uma intensa deformação plástica. A eficiência da tenacificação depende em geral do tipo específico do modificador empregado, tamanho e distribuição das partículas, temperatura de transição vítrea e compatibilidade entre as fases [12].

O SBR (copolímero de estireno-butadieno) é um elastômero que possui propriedades como boa resistência ao envelhecimento, à abrasão e à água do mar [13]. No desenvolvimento de formulações para compostos elastoméricos utiliza-se um grande número de aditivos como cargas, agente de cura, ativador, acelerador, retardador, auxiliar de processo, plastificante, antidegradante ou antioxidante, que ajudam a proteger a borracha contra os efeitos da deterioração por envelhecimento, exposição à luz solar e ozônio e outras substâncias com funções mais específicas como, por exemplo, retardante de chama, agentes de expansão, dentre outros. Cada um desses componentes é importante tanto nas propriedades quanto no custo da formulação [14]].

A maioria das misturas de PS/elastômeros é imiscível, ou seja, existe segregação de fases. Em misturas imiscíveis, as propriedades finais são resultados da contribuição individual de cada um dos componentes [15]. O desenvolvimento de misturas imiscíveis tem como resultado a obtenção de propriedades limitadas e até inferiores aos componentes poliméricos puros. Que neste caso são consideradas incompatíveis e necessita da adição de um agente compatibilizante para melhorar a interação entre as fases e tornar a blenda compatível em seu estado de mistura [16]. A adição adequada de compatibilizantes nas misturas imiscíveis deverá: reduzir a energia interfacial entre as fases; melhorar a adesão interfacial; permitir uma dispersão e prover a estabilidade da mistura [1-18].

Este trabalho teve o objetivo de produzir misturas de PS/SBR, utilizando um copolímero em bloco como agente compatibilizante, visando à obtenção de materiais com propriedades superiores ao poliestireno.

\section{MATERIAIS E MÉTODOS}

\subsection{Materiais}

Matriz polimérica 
Foi utilizado poliestireno cristal (PS) sob o código 158K Q611 na forma de grânulos, fabricado pela Unigel S.A., que apresenta como característica principal excelente propriedade de brilho e transparência e é adequado à moldagem por extrusão e injeção.

\section{Fase dispersa}

Foi utilizado um resíduo de borracha reciclada (SBR), proveniente da indústria de calçados São Paulo Alpargatas S.A (Sandálias Havaianas) da região de Campina Grande/PB.

\section{Compatibilizante}

Foi utilizado o copolímero tribloco linear (SBS), sob o código Kraton D1101B, a base de estireno e butadieno contendo 31\% de poliestireno, na forma de grânulos, adquirido da Activas S.A.

\subsection{Métodos}

\section{Preparação das blendas}

As misturas binárias PS/SBR foram preparadas nas proporções em peso de 70/30, 60/40 e 50/50\% e as ternárias PS/SBR/SBS nas proporções em peso de 67,5/27,5/5; 57,5/37,5/5 e 47,5/47,5/5\%. A fase dispersa passou em uma peneira ABNT No $40(0,420 \mathrm{~mm})$.

As misturas foram processadas em uma extrusora dupla rosca corrotacional da Coperion ZSK $18 \mathrm{~mm}$ com temperatura de $190^{\circ} \mathrm{C}$ nas zonas 1 e 2 e $200^{\circ} \mathrm{C}$ nas demais zonas; velocidade de $250 \mathrm{rpm}$ e taxa de alimentação de $4 \mathrm{~kg} / \mathrm{h}$. Os grânulos extrusados foram secos, numa estufa a vácuo com temperatura de $60^{\circ} \mathrm{C}$ durante 48 horas.

Após a secagem, os grânulos extrusados foram moldados por injeção na forma de corpos de prova para ensaios de DMTA (Corpos de prova tipo Izod ASTM D256), tração (ASTM D638) e HDT (ASTM D648). Os corpos de prova foram moldados numa temperatura de $180^{\circ} \mathrm{C}$ na zona 1 e $190^{\circ} \mathrm{C}$ na zona 2 , tendo um ciclo de injeção de $25 \mathrm{~s}$ e temperatura do molde de $40^{\circ} \mathrm{C}$.

\section{TÉCNICAS DE CARACTERIZAÇÃO}

\section{Ensaio sob tração}

Os testes de resistência à tração uniaxial foram realizados em corpos de prova injetados, segundo a norma ASTM D638, utilizando uma máquina de ensaios universal da marca EMIC DL 2000, com velocidade de carregamento de $5 \mathrm{~mm} / \mathrm{min}$. Os testes foram conduzidos a temperatura ambiente e os resultados analisados com uma média de 10 corpos de prova.

\section{Ensaio de temperatura de deflexão térmica (HDT)}

A temperatura de deflexão térmica (HDT) foi obtida, conforme a norma ASTM D648, em um equipamento Ceast, modelo HDT 6 VICAT, com uma tensão de 1,82 MPa, taxa de aquecimento de $120^{\circ} \mathrm{C} / \mathrm{h}$ (método A). A temperatura foi determinada após a amostra ter defletido $0,25 \mathrm{~mm}$. Uma série de seis amostras foi ensaiada e a temperatura de deflexão térmica medida.

\section{Análise térmica dinâmica mecânica (DMTA)}

A análise térmica dinâmico-mecânica (DMTA) foi realizada em um equipamento de DMTA da marca TA Instruments Explorer, modelo Q 800. O modo de solicitação dinâmico-mecânico utilizado foi o de flexão em um ponto, adequado para os corpos de prova tipo Izod utilizados nos ensaios. A taxa de aquecimento utilizada foi de $10^{\circ} \mathrm{C} / \mathrm{min}$ e frequencia de $1 \mathrm{~Hz}$, na faixa de temperatura de -100 a $150^{\circ} \mathrm{C}$.

\section{Microscopia eletrônica de varredura (MEV)}

As análises por microscopia eletrônica de varredura (MEV) foram realizadas na superfície de fratura dos corpos de prova submetidos ao ensaio sob impacto em um microscópio eletrônico de varredura, Shimadzu SSX-550 Superscan, a uma voltagem de $15 \mathrm{kV}$, sob alto vácuo e as superfícies de fratura recobertas com ouro. 


\section{RESULTADOS E DISCUSSÃO}

\subsection{Ensaio sob tração}

As Figuras 1 e 2 mostram os valores das propriedades mecânicas obtidas por ensaios de tração do PS, das misturas binárias e ternárias. Observa-se nas Figuras 1 e 2 que todos os valores do módulo de elasticidade e da resistência à tração foram abaixo daqueles obtidos para o PS. Isto estaria relacionado à estrutura química do PS que possui anéis aromáticos em sua cadeia lateral que conferem rigidez a este polímero tornando-o vítreo [므-20].

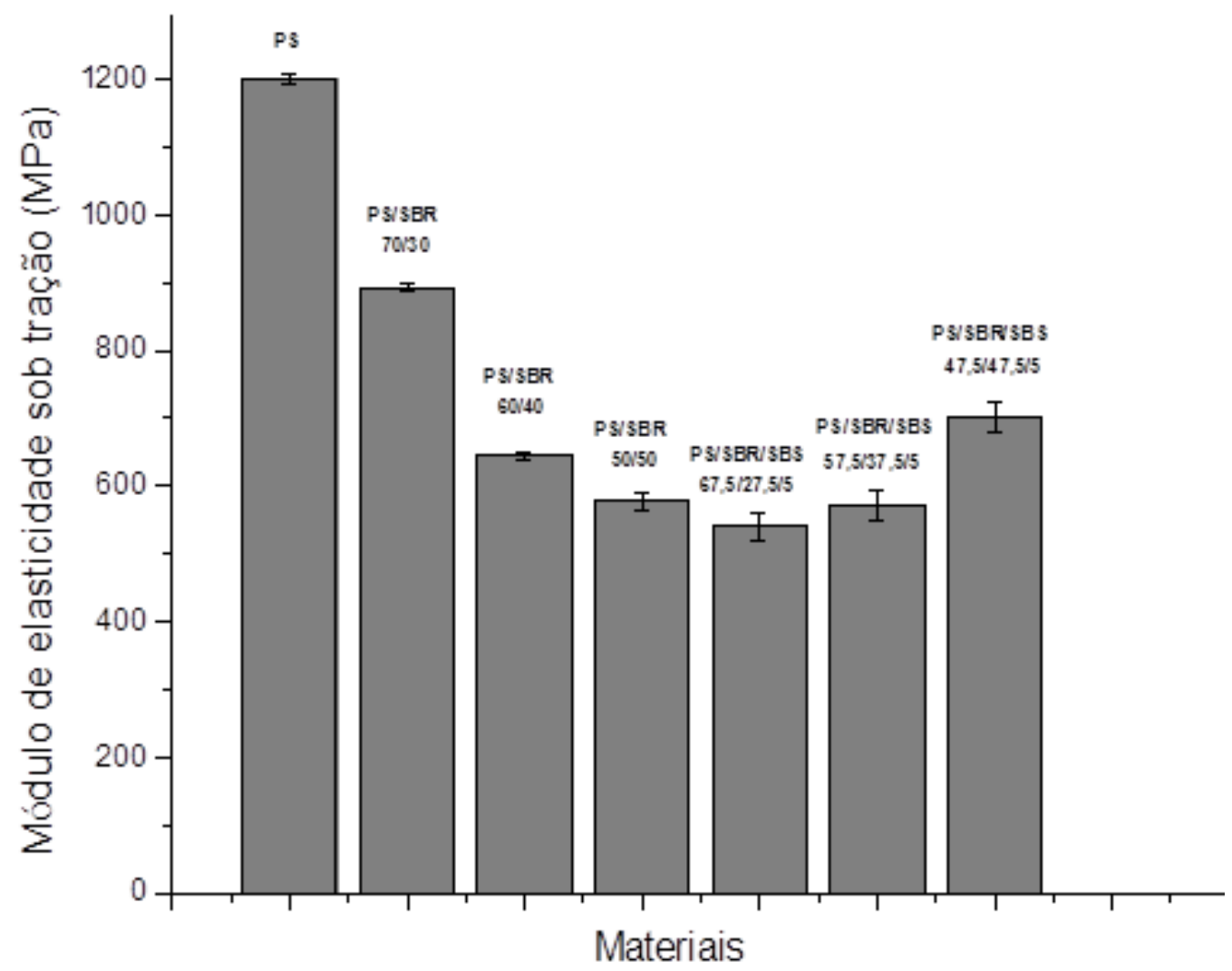

Figura 1: Módulo de elasticidade sob tração do PS, das misturas binárias e ternárias 


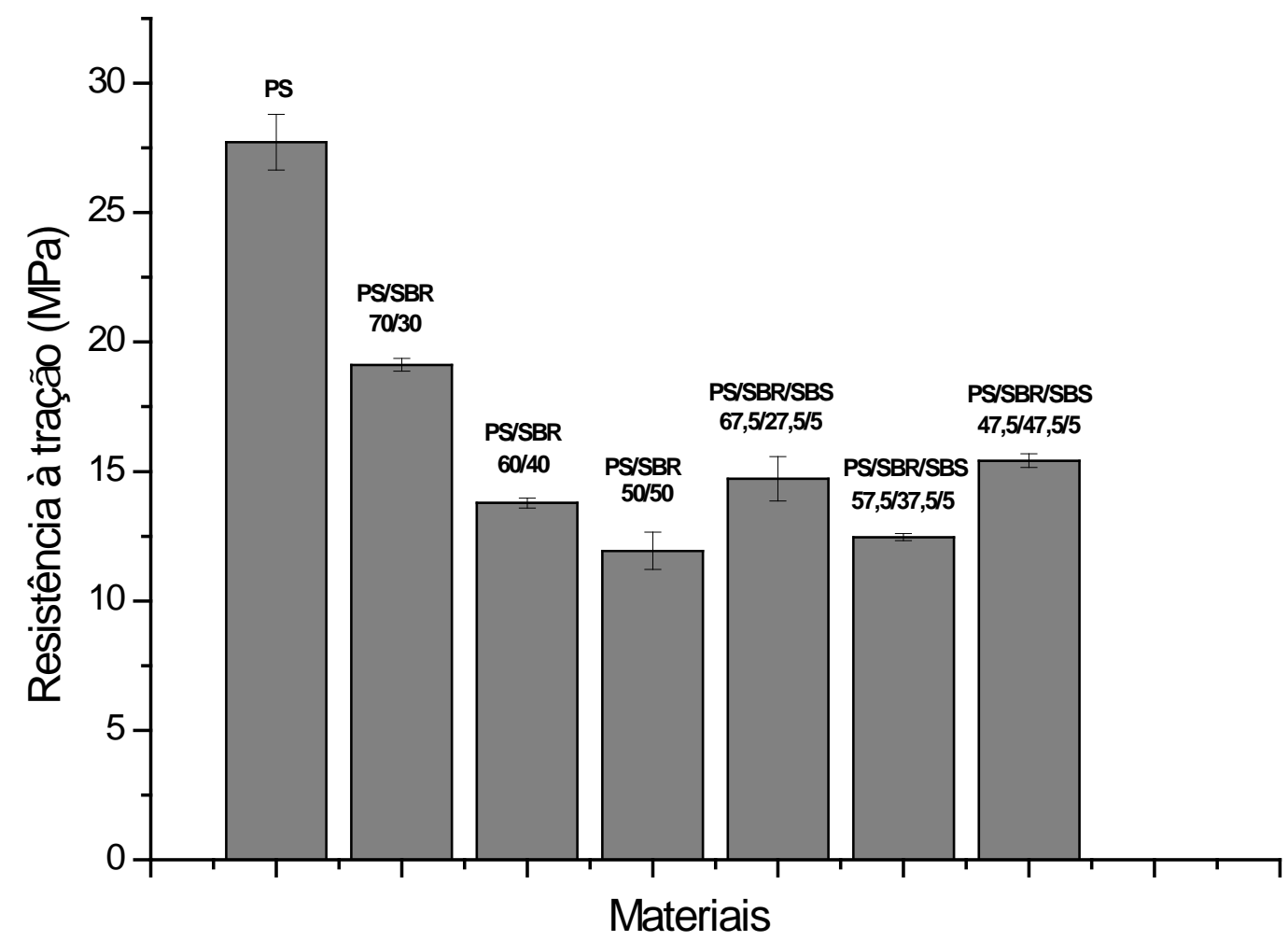

Figura 2: Resistência á tração do PS, das misturas binárias e ternárias.

Observa-se na Figura 2 que a adição de um material flexível a uma matriz rígida e amorfa provoca uma redução na resistência à tração nas misturas binárias (PS/SBR), e que estas variações são mais acentuadas com o aumento do teor de borracha incorporada. Isto poderia ser atribuído à baixa interação física do pó de borracha com a matriz de PS, que ocasiona uma fraca interação interfacial entre eles [21] .

MELO et al.[16], reportaram que à medida que aumenta o teor de elastômero na mistura diminui a interação das fases PS e SBR, o que pode enfraquecer a interface do sistema e dificultar a transferência de tensões entre as fases diminuindo a resistência da mistura.

Conforme BOONDAMNOEN et al.[22], a proporção de borracha afeta as propriedades mecânicas das misturas. Eles afirmam que a resistência à tração e o módulo de elasticidade tendem a diminuir quando se aumenta a fração de borracha, uma vez que o aumento de borracha reduz a rigidez da mistura.

Por outro lado, quando se considera que estamos utilizando um resíduo industrial que apresenta uma mistura complexa de SBR, cargas, aditivos de processamento, agentes de cura e estabilizantes, esta queda de propriedades é aceitável uma vez que estamos perdendo no máximo 50\% destas propriedades sob tração para a blenda na proporção de 50/50\% e aumentando em $112 \%$ na tenacidade da mesma mistura, como se observa no trabalho de LUNA et al. [23]. Além disso, pode-se reduzir custo uma vez que estamos utilizando um resíduo descartado, bem como diminuir os impactos destes rejeitos no meio ambiente.

Nas Figuras 1 e 2 verifica-se também os resultados das misturas ternárias PS/SBR/SBS com 5\% de compatibilizante SBS. Nota-se que à medida que aumenta o teor de borracha nas misturas ternárias e mantém constante o teor de SBS, é verificado um efeito positivo em relação ao módulo de elasticidade com um aumento gradativo. Porém nota-se uma oscilação na resistência à tração. Este decaimento na resistência à tração na mistura 57,5/37,5/5 \% possivelmente é causado por zonas de concentração de tensão que acelera o processo de ruptura do material sob tração. Já para a blenda 47,5/47,5/5\% ocorreu um aumento moderado na resistência à tração em relação à blenda 67,5/27,5/5 \%. Isto provavelmente é devido ao aumento da força de adesão entre as fases PS e SBR [24].

De uma maneira geral, a mistura ternária 47,5/47,5/5\% apresentou resultados superiores em relação às outras misturas ternárias. BOONSONG et. al.[25] afirmam que essa melhoria é devido a uma maior adesão entre os limites das fases constituintes dessa blenda compatibilizada. Isto aumenta o nível de tolerância de tensão, que é transferida através da interface da mistura. 
De acordo com SANGWICHIEN et. al.[26], o valor superior na mistura 47,5/47,5/5\% em relação 67,5/27,5/5\% e 57,5/37,5/5\%, possivelmente foi devido ao aumento da adesão interfacial que suprimiu a produção de vazios.

O aumento do módulo de elasticidade e da tensão máxima no escoamento nas misturas compatibilizadas é tomado como um indicativo que a adição do SBS promove a interação entre as fases presentes, fortalecendo a interface do sistema, o que é fundamental para a transferência de tensões entre as fases. Porém, a falta de interação acarreta a presença de microvazios nas interfaces, o que leva à formação de trincas e, facilita a propagação destas, resultando em baixa resistência sob tração como se observa na mistura PS/SBR/SBS na proporção 57,7/37,5/5\% [1] .

\subsection{Ensaio de temperatura de deflexão térmica (HDT)}

O ensaio de temperatura de deflexão térmica é de fundamental importância em polímeros. A partir dele podese determinar uma temperatura máxima de trabalho para um dado polímero, acima da qual o material possivelmente não conseguirá desempenhar as funções para a qual foi projetado [27]. ensaiados.

A Figura 3 ilustra o comportamento obtido para a temperatura de deflexão térmica dos materiais

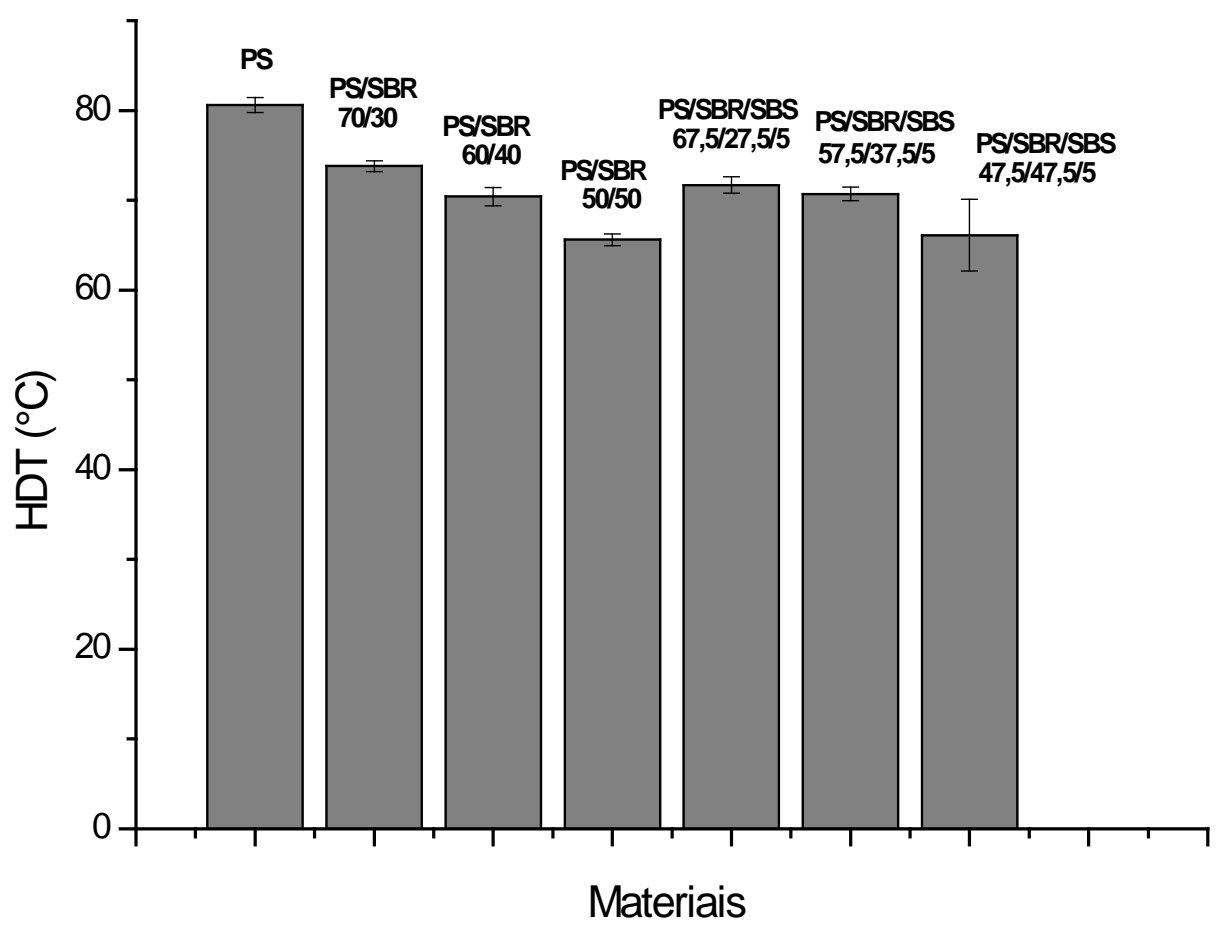

Figura 3: Temperatura de deflexão térmica do PS, das misturas binárias e ternárias.

Verifica-se pela análise da Figura 3que a HDT do PS ficou na faixa de $80^{\circ} \mathrm{C}$. Isto pode ser atribuído ao fato do PS ser caracterizado como um material rígido e, possivelmente necessita de uma maior temperatura para que o corpo de prova sofra deflexão. Analisando-se os resultados da Figura 3, no geral, todas as misturas apresentaram uma temperatura de deflexão térmica abaixo do PS, independente de ser compatibilizada ou não. Provavelmente a incorporação do SBR ao PS, contribuiu para a diminuição da HDT. Entretanto, quando se considera que a incorporação de 50 e $47,5 \%$ em peso de um material reciclado ao PS, esta redução é aceitável já que não compromete significativamente as propriedades e contribui para a redução de custo do composto final. 
A temperatura de deflexão térmica não sofre influência com a variação da maioria das variáveis, mas somente com o aumento do teor de borracha. Afinal, o HDT é uma propriedade que depende mais significativamente da fase contínua, que é responsável pela rigidez do material [28]. Observando-se a Figura 3 , nota-se que as misturas binárias e ternárias ratificam a teoria, ou seja, o teor de elastômero influenciou a temperatura de deflexão térmica. Nota-se que as misturas tiveram uma queda da HDT com o aumento do teor de elastômero. Provavelmente este decaimento com o aumento do teor de borracha é devido ao fato de terem se tornados mais flexíveis com a presença do elastômero, necessitando, assim de uma menor temperatura para que o corpo de prova sofra deflexão.

As misturas ternárias 67,5/27,5/5 e 57,5/37,5/5\% praticamente tiveram o mesmo comportamento em relação à temperatura de deflexão térmica. As composições 47,5/47,5/5\% e 50/50\% das misturas ternárias e binárias, respectivamente, tiveram os mais baixos desempenhos da HDT. Isto pode ser atribuído a maior quantidade de elastômero nessas misturas, tornando-as mais flexíveis. Resultados similares foram encontrados por Pereira et al. [29], na ocasião eles produziram misturas de PP/resíduos de pneus. Estes autores processaram as misturas nas proporções de 100/0\%; 90/10\%; 80/20\% e 70/30\% em peso. Ficou evidenciado que à medida que aumentou o teor de borracha na matriz polimérica houve o decaimento da propriedade de HDT. Portanto, possivelmente o alto teor de borracha incorporada nas misturas de PS/SBR deste trabalho foi o fator preponderante para perda desta propriedade.

A mistura binária 70/30\% foi a que obteve o melhor resultado de HDT, aproximado ao valor do PS. Uma possível explicação para esse fato, segundo estudos de FERREIRA et al.[30], é que as características mais importantes no desempenho de uma mistura sob temperatura de deflexão térmica são a contribuição individual dos componentes e a morfologia gerada pelas fases nas misturas. Geralmente, a fase contínua numa morfologia de fases proporciona maior contribuição a HDT da blenda polimérica. A fase contínua, na maioria das vezes, é a própria matriz da mistura. Então, provavelmente o PS como uma matriz rígida contribuiu mais significativamente na temperatura de deflexão térmica do que o SBR nesta mistura 70/30\%. O valor observado nesta mistura é muito interessante do ponto de vista tecnológico, uma vez que está próximo ao PS, ou seja, não houve uma grande perda da HDT.

\subsection{Análise térmica dinâmico mecânica (DMTA)}

Por meio da análise térmica dinâmico-mecânica (DMTA), pode-se estimar mudanças de relaxações moleculares que ocorrem em materiais poliméricos em uma ampla faixa de temperatura. O conhecimento dessas mudanças permite relacionar parâmetros moleculares e propriedades mecânicas de polímeros.

As Figuras 4, 5 e 6 representam a tangente de perda, o módulo de perda e o módulo de armazenamento respectivamente, do Poliestireno, da mistura binária 50/50\% e ternária 47,5/47,5/5\%. Esses materiais foram escolhidos, pois apresentaram os melhores resultados no processo de tenacificação como reportado por LUNA et al. [르].

A Figura 4 apresenta os valores da tangente de perda, tan ( $\delta$ ), do PS, da mistura binária 50/50\% e da sequência de mistura PS/SBR/SBS 47,5/47,5/5\%. Observa-se na Figura 4, a presença de dois picos de transição bem distintos: um a aproximadamente $112^{\circ} \mathrm{C}$, relacionado à temperatura de transição vítrea (Tg) do PS e a, aproximadamente $-36^{\circ} \mathrm{C}$, referente à fase elastomérica presente no SBR. Para a mistura binária e a sequência de mistura houve deslocamento do pico da Tg do PS para valores menores de aproximadamente $101^{\circ} \mathrm{C}$. Isto pode ser devido à influência da presença do elastômero SBR na blenda que apresenta uma Tg bem abaixo da Tg do PS. Segundo QUENTAL et al.[31], em seu estudos, relataram que misturas parcialmente miscíveis apresentam mais de uma transição vítrea, porém as temperaturas estão deslocadas em relação às Tg dos componentes puros. Então sugere-se que as blendas de PS/SBR e PS/SBR/SBS apresentam características de blendas parcialmente miscíveis. 


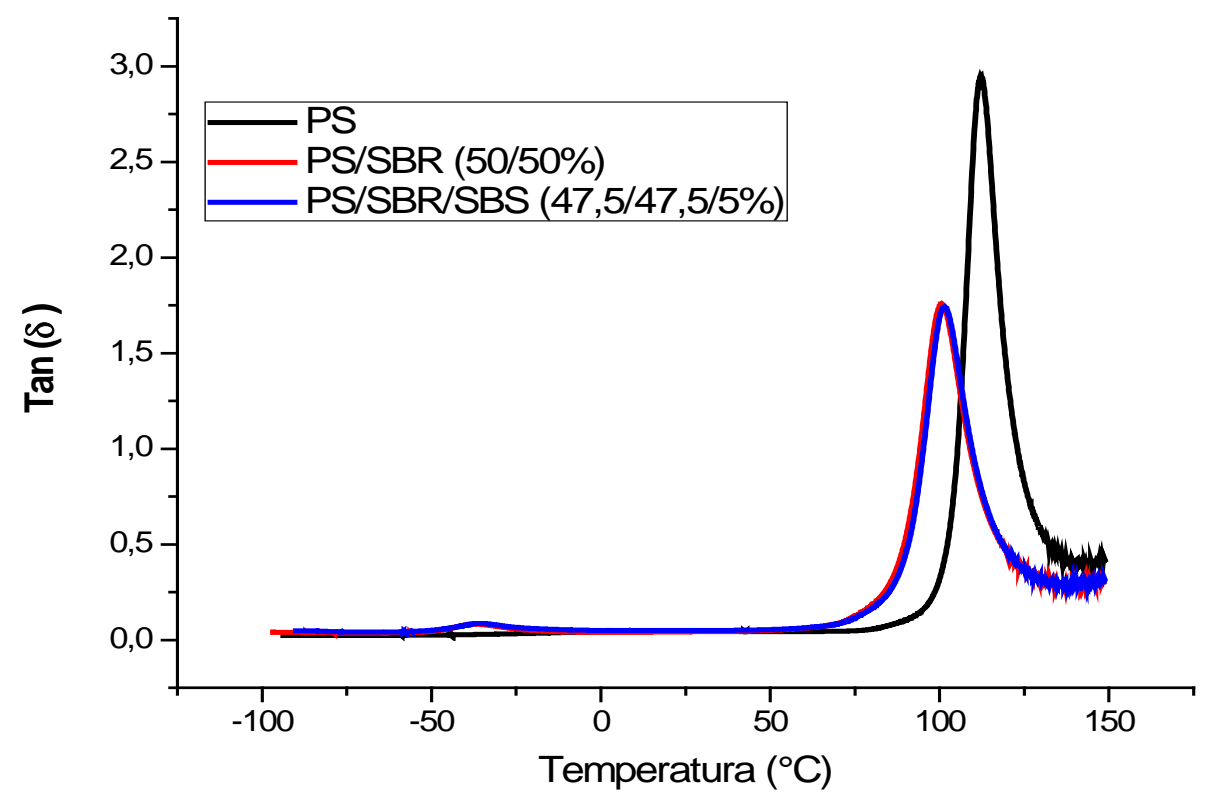

Figura 4: Curvas da tangente de perda tan $(\delta)$ em função da temperatura para o PS, da mistura binária PS/SBR (50/50\%) e ternária PS/SBR/SBS (47,5/47,5/5\%).

A Figura 5 apresenta os valores de módulo de perda (E”) do PS, da mistura binária 50/50\% e da PS/SBR/SBS 47,5/47,5/5\%.

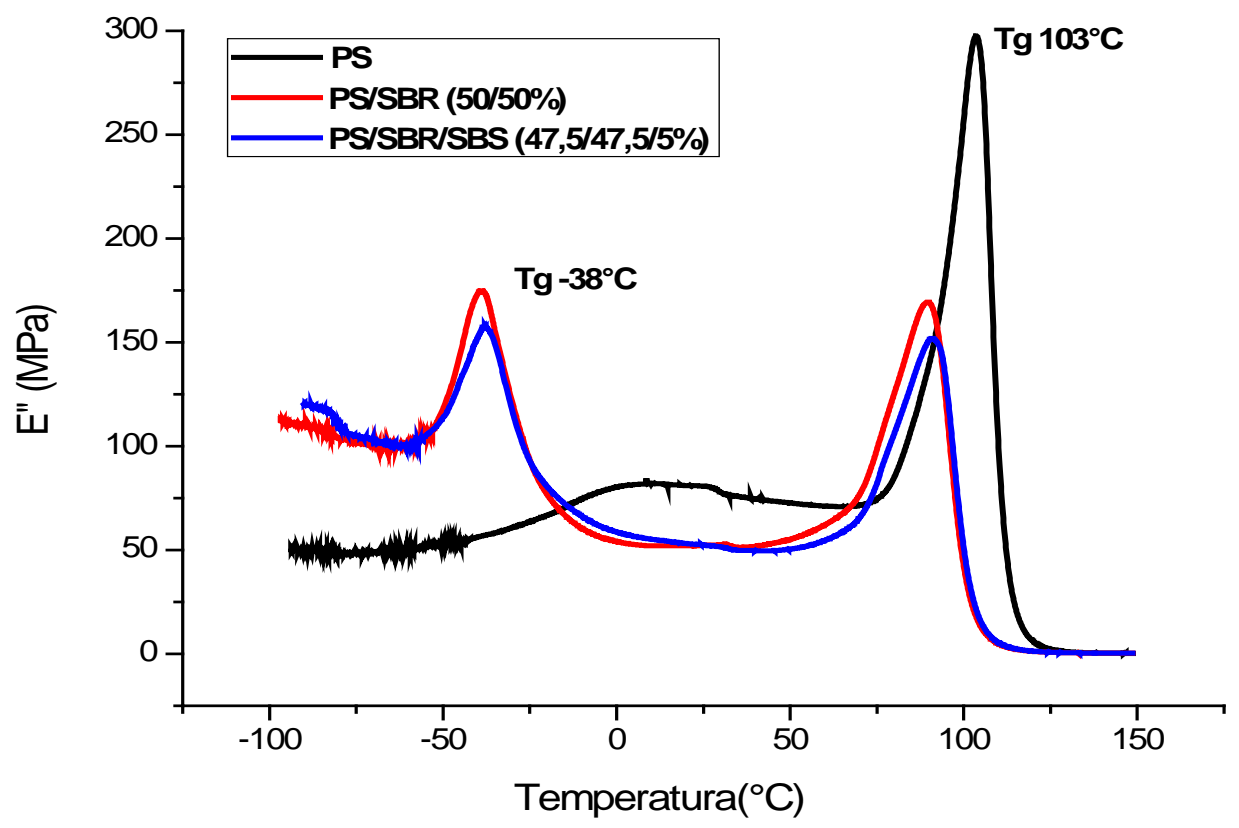

Figura 5: Curvas do módulo de perda (E”) em função da temperatura para o PS, a mistura binária (PS/SBR) e para a mistura PS/SBR/SBS (47,5/47,5/5\%).

Observa-se na Figura 5 que o PS apresenta um pico em aproximadamente $103^{\circ} \mathrm{C}$, relacionado à sua $\mathrm{Tg}$ e, em $-38^{\circ} \mathrm{C}$, relacionado à $\mathrm{Tg}$ da fase do polibutadieno presente no SBR. Para a mistura binária e a ternária 
houve deslocamento dos picos para aproximadamente $101^{\circ} \mathrm{C}$. Não se observou mudança significativa na transição vítrea das misturas de PS/SBR e da sequência PS/SBR/SBS (47,5/47,5/5\%), indicando que o compatibilizante SBS e a sequência de mistura não influenciaram na Tg desses sistemas, mas com relação ao PS houve deslocamento dos picos da Tg's.

O máximo do pico da curva corresponde à situação de máxima dissipação de energia mecânica, que na região de transição vítrea está associada à mudança do estado vítreo para o elástico. É uma característica de todos os dados dinâmicos mecânicos que o pico de tan $(\delta)$ não apareça no mesmo ponto do módulo de perda. Uma vez que o fator de amortecimento pode ser interpretado matematicamente como sendo a variável que define a taxa de mudança do módulo de elasticidade, ela deve ser utilizada para a determinação de temperaturas de transição. Em materiais amorfos, o valor de tan $(\delta)$ normalmente excede a unidade, indicando que o comportamento viscoso é dominante [32].

A Figura 6 apresenta os valores de módulo de armazenamento (E’) do PS, da mistura binária 50/50\% e da PS/SBR/SBS 47,5/47,5/5\%.

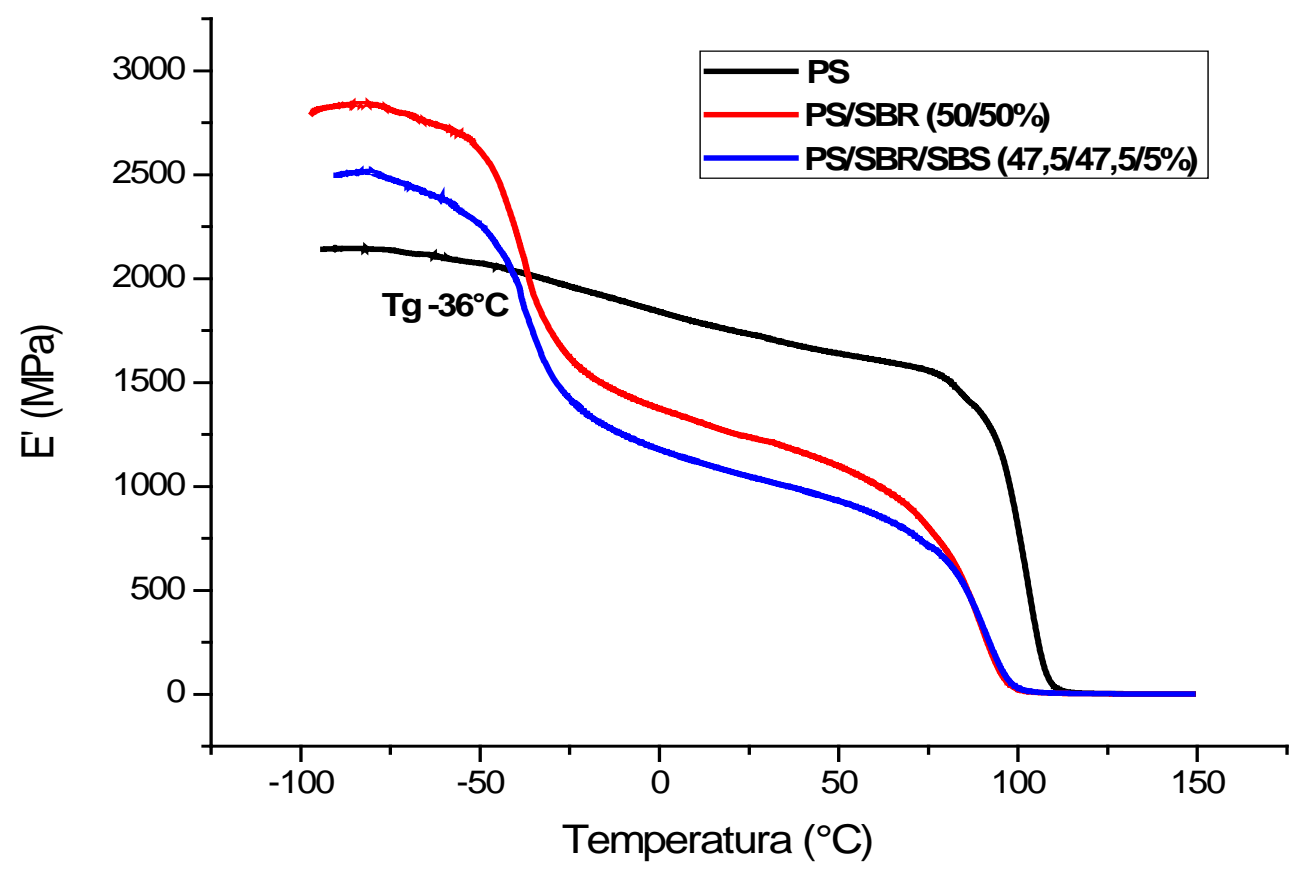

Figura 6: Curvas do módulo de armazenamento (E’) em função da temperatura para o PS, a mistura binária (PS/SBR) e para a mistura PS/SBR/SBS (47,5/47,5/5\%).

Na Figura 6 todas as curvas apresentaram o mesmo comportamento dinâmico-mecânico, típico de polímeros amorfos, em função da temperatura. A transição vítrea é caracterizada pela queda abrupta do módulo de armazenamento (E'), característica de polímeros amorfos. Nas curvas relativas às misturas poliméricas, observa-se uma pequena queda inicial, em torno de $-36^{\circ} \mathrm{C}$, que se deve à transição vítrea da fase elastomérica. Observa-se uma queda mais acentuada na sequência de mistura PS/SBR/SBS (47,5/47,5/5\%), caracterizando uma tendência a uma melhor interação desse elastômero com a matriz PS.

Na Figura 6 observa-se um plateau que determina o módulo de armazenamento dos polímeros. Na faixa de aplicação da mistura que é acima da Tg da fase polibutadieno do SBR e abaixo da Tg do PS, as misturas com elastômero baixam o módulo de armazenamento da matriz PS, isto ocorre devido à presença da fase elastómerica que dissipa rapidamente a energia mecânica recebida. Pode-se notar outro indicativo de miscibilidade parcial, pois os módulos são diferentes para cada amostra.

\subsection{Microscopia eletrônica de varredura}


Nas Figuras 7 a 9 estão apresentadas as fotomicrografias obtidas por MEV com um aumento de 1000x do PS puro, da mistura binária 50/50\% e ternária 47,5/47,5/5\%. Estas amostras apresentaram melhores desempenhos na resistência ao impacto conforme estudos de LUNA et al. [23]. Estas fotomicrografias foram obtidas da superfície de fratura dos corpos de prova submetidas ao ensaio de impacto Izod.

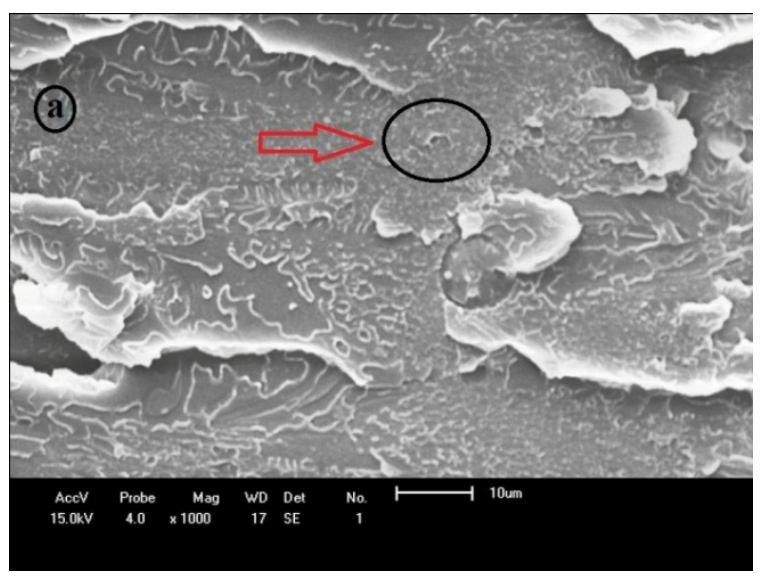

Figura 7: Fotomicrografia do PS.

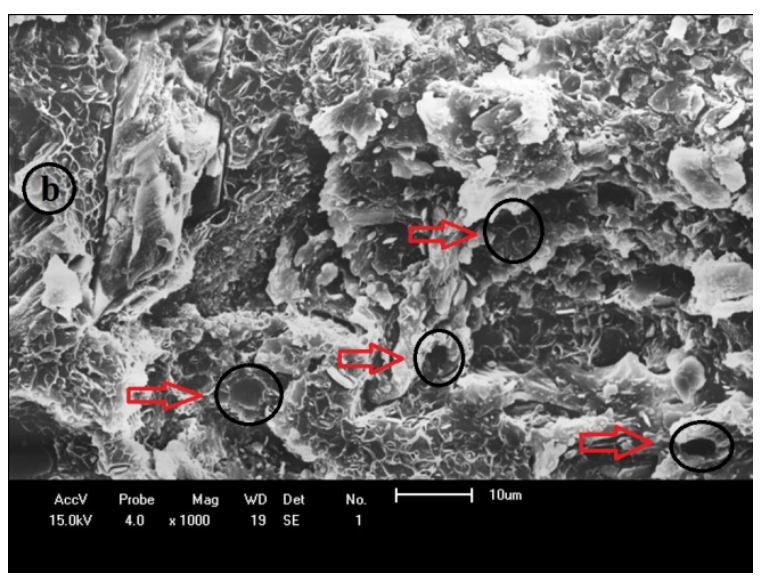

Figura 8: Fotomicrografia da mistura 50/50\%.

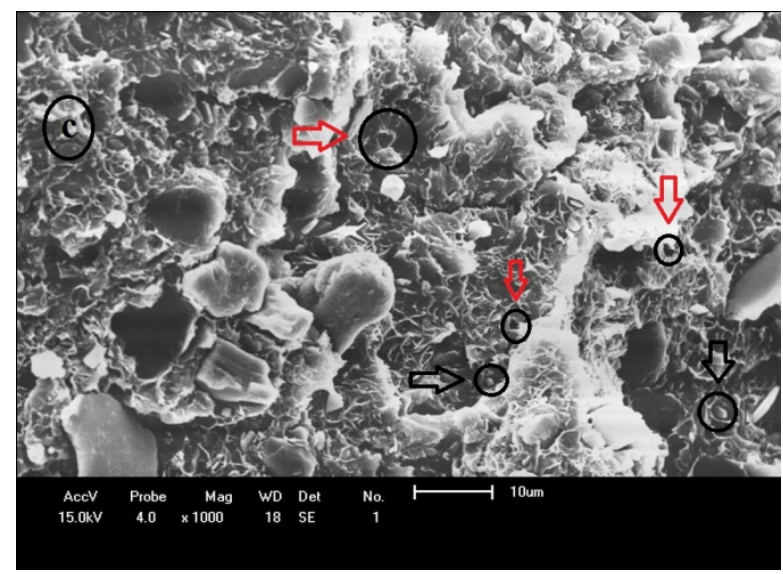

Figura 9: Fotomicrografia da mistura 47,5/47,5/5\%.

A Figura 7 apresenta a fotomicrografia do PS puro, no qual apresenta um aspecto liso e homogêneo, que está associado à natureza frágil deste polímero de baixa tenacidade. Além disso, observam-se estrias correspondentes as marcas e degraus deixados pela trinca responsável pela ruptura do corpo de prova e que foi nucleada a partir do entalhe [33]. Esta trinca que se propagou deixou marcas envolvendo pequenas 
cavidades (seta vermelha). Na fotomicrografia 7, pode-se constatar que praticamente não existe deformação plástica, bem como característica de uma trinca instável no qual se propaga rapidamente.

Na Figura 8, pode-se observar a má adesão entre as fases, provavelmente devido à fraca interação interfacial entre estas, bem como, a presença de vazios (seta vermelha) entre os domínios de borracha e a matriz. A morfologia irregular e as superfícies bem definidas dos vazios indicam baixa interação interfacial entre os dois polímeros. Por meio da morfologia da superfície de fratura da Figura 8, pode-se ratificar os resultados das Figuras 1,2 e 3, os quais apresentaram propriedades que foram inferiores em relação às dos sistemas compatibilizados. Além disso, esse sistema apresentou menor resistência ao impacto em relação às misturas compatibilizadas conforme pode averiguar no trabalho de LUNA et al. [23].

Como se pode constatar na Figura 9, a adição do agente compatibilizante na mistura promoveu uma melhor adesão interfacial entre os domínios e a matriz, bem como alterou o tamanho dos domínios. Como reportado na literatura [34-35-36], o compatibilizante tende a reduzir o tamanho das partículas. Nota-se que $5 \%$ do compatibilizante SBS promoveu uma diminuição dos domínios das partículas de borracha (seta preta) e dos vazios (seta vermelha) em relação à mistura binária (Figura 8). Então, a redução dos tamanhos das partículas dispersas provavelmente foi o fator responsável pelo aumento das propriedades em relação ao sistema não compatibilizado. Além disso, observa-se partículas parcialmente recobertas pela matriz. Este fato é de grande importância, pois evidencia que a adesão matriz/compatibilizante/partículas de borracha desta mistura foi boa.

\section{CONCLUSÕES}

Misturas de PS/composto de borracha reciclada foram produzidas. Os resultados do ensaio de tração evidenciaram que, no geral, o módulo de elasticidade de todas as blendas reduziu quando comparado ao PS, o que pode ser atribuído ao fato da adição de uma fase dispersa flexível e tenaz como o SBR. Com relação à temperatura de deflexão térmica, a mistura binária $70 / 30 \%$ foi a mais interessante do ponto de vista tecnológico, uma vez que o valor obtido está próximo ao PS e não houve uma grande perda da HDT. A análise termomecânica de DMTA indicou uma miscibilidade parcial do sistema PS/composto de borracha reciclada. A morfologia do MEV evidenciou que o compatibilizante reduziu de forma significativa os tamanhos dos vazios, bem como o tamanho da fase dispersa. Portanto, os resultados evidenciam que misturas de PS/composto de borracha reciclada obtidas por meio de misturas mecânicas podem ser adequadas do ponto de vista tecnológico e também proporcionam uma boa perspectiva de aplicação destes rejeitos industriais, uma vez que podem reduzir os efeitos nocivos sobre o meio ambiente e agregar valor a um produto que seria descartado. Sabe-se que apenas $40 \%$ destes resíduos são reaproveitados na empresa e que o restante é incinerado. Além disso, as misturas poliméricas podem proporcionar a obtenção de um material com propriedades boas.

\section{AGRADECIMENTOS}

Os autores agradecem a CAPES (Coordenação de Aperfeiçoamento de Pessoal de Nível Superior) pela concessão de bolsa de estudo do doutorado e ao PIBIC, bem como, a São Paulo Alpargatas/PB pelo fornecimento das matérias primas, ao MCTI/CNPq e a CAPES/PNPD.

\section{BIBLIOGRAFIA}

[1] CHUAYJULJIT, S., MOOLSIN, S., POTIYARAJ, P., "Use of natural rubber-g-polystyrene as a compatibilizer in casting natural rubber/polystyrene blends films", Journal of Applied Polymer Science, v. 95, n. 4, pp. 826-831, 2005.

[2] FERNANDES, L. L., FREITAS, C. A., DEMARQUETTE, N. R., et al., "Estudo do efeito do tipo de polipropileno na fotodegradação da blenda polipropileno/poliestireno de alto impacto", Polímeros: Ciência e Tecnologia, v.22, n.1, pp.61-68, 2012.

[3] NASCimentO, W. A., LIMA, J. C. C., AGRAWAL, P., et al., "Blendas de PP/PA6: efeito da concentração de PA6 e compatibilizantes reativos nas propriedades mecânicas, térmicas e morfológicas", Revista Eletrônica de Materiais e Processos, v.8, n.3, pp.155-162, 2013.

[4] RIBEIRO, V. F., Estudo da recuperação das propriedades mecânicas de poliestireno alto impacto reciclado através da incorporação de borrachas termoplástica tipo estireno-butadieno-estireno, TCC de Graduação., CCT/Feevale, Novo Hamburgo, RS, Brasil, 2008.

[5] BLAZEK, G. R., Estudo da blenda poli(3hidroxibutirato)/poli(etilenoglicol), Dissertação de M.Sc., CCT/USP, São Paulo, SP, Brasil, 2012. 
[6] DAGLI, S. S., XANTHOS, M., BIESENBERGER, J. A., "Kinetic studies and process analysis of reactive compatibilization of nylon 6/polypropylene blends”, Polymer Engineering and Science, v. 34, n. 23, pp. 1720-1730, 1994.

[7] VERONESE, V. B., Desenvolvimento e avaliação de misturas de poliestireno (PS) e copolímero em bloco estireno-butadieno-estireno (SBS), Dissertação de M.Sc., CCT/UFRGS, Porto Alegre, RS, Brasil, 2003.

[8] COUTINHO, F. M. B., COSTA, M. P. M., GUIMARÃES, M. J. O. C., et al., "Estudo comparativo de diferentes tipos de polibutadieno na tenacificação de poliestireno”, Polímeros: Ciência e Tecnologia, v.17, n.4, pp.318-324, 2007.

[9] WANG, Z., CHAN, C. M., ZHU, S. H., et al., Compatibilization of polystyrene and low density polyethylene blends by a two-step crosslinking process”, Polymer, v. 39, n. 26, pp. 6801-6806, 1998.

[10] LEITZKE, T. C., PEZZIN, A. P. T., PEZZIN, S. H., "Blendas SAN/NBR: Influência do teor de acrilonitrila e da viscosidade da borracha nitrílica nas propriedades mecânicas”, Revista Matéria, v. 12, n. 4, pp. 568-573, 2007.

[11] PIZZOL, M. F. D., GRASSI, V. G., “Caracterização morfológica do poliestireno de alto impacto (HIPS)”,Polímeros: Ciência e Tecnologia, v.18, n.1, pp.12-19, 2008.

[12] GRASSI, V. G., FORTE, M. M. C., “Aspectos morfológicos e relação estrutura propriedades de poliestireno de alto impacto”, Polímeros: Ciência e Tecnologia, v.11, n.3, pp.158-168, 2001.

[13] SILVA, D. F., ARAÚJO, E. M., MELO, T. J. A., "Desenvolvimento de blendas de poliamida 6/composto de borracha reciclada (SBRr)”,Revista Eletrônica de Materiais e Processos, v.7, n.2, pp.104-110, 2012.

[14] SERRANO C. L., R., Uso de resíduos de SBR proveniente da indústria de calçados em composição de SBR, Tese de D.Sc., CCT/UFRGS, Porto Alegre, RS, Brasil, 2009.

[15] CIGANA, P., FAVIS, B. D., JEROME, R., "Diblock copolymers as emulsifying agents in polymer blends: influence of molecular weight, architecture and chemical composition”, Journal Polymer Science: Part B: Polymer Physics, v, 34, n.9, pp. 1691-1700, 1996.

[16] MÉLO, T. J. A., CALUMBY, R. B.,BRITO, K. G. Q., et al., “ Propriedades mecânicas e morfológicas de uma blenda polimérica de PP/HIPS compatibilizada com SEBS”, Polímeros: Ciência e Tecnologia, v.10, n.2, pp.82-89, 2000.

[17] HO, C-H., WANG, C-H., LIN, C-I., et al., "Synthesis and characterization of TPO-PLA copolymer and its behavior as compatibilizer for PLA/TPO blends”, Polymer, v. 49, n. 18, pp. 3902-3910, 2008.

[18] LUNA, C. B. B., SILVA, D. F., ARAÚJO, E. M., “Análise do comportamento termomecânico, térmico e mecânico de blendas de PA6/resíduos de borracha”, Revista de Engenharia e Tecnologia, v. 6, n.1, pp. 160169, 2014.

[19] GILBERT, D. G., DONALD, A. M., “Toughening mechanisms in high impact polystyrene”, Journal of Materials Science, v. 21, n. 5, pp. 1819-1823, 1986.

[20] YOKOUCHI, M., UCHIYAMA, H., KOBAYASHI, Y., "Effect of tensile strain rate on the mechanical properties of polystyrene and high-impact polystyrene”, Journal of Applied Polymer Science, v. 25, n. 6, pp.1007-1015, 1980.

[21] SONNIER, R., LEROY, E., CLERC, L., et al., "Polyethylene/ground tyre rubber blends: influence of particle morphology and oxidation on mechanical properties”, Polymer Testing: Material Properties, vol.26, n.2, pp. 274-281, 2007.

[22] BOONDAMNOEN, O., OHSHIMA, M., AZURA. A.R., et al., "Recycling waste natural rubber latex by blending with polystyrene characterization of mechanical properties”, International Journal of Modern Physics: Conference Series, v.6, n.2, pp. 391-396, 2012.

[23] LUNA, C. B. B., SILVA, D. F., ARAÚJO, E. M., "Reaproveitamento dos resíduos de borracha em blendas com poliestireno”, Estudos Tecnológicos em Engenharia, vol. 10, n. 1, pp. 1-9, 2014.

[24] GREIN, C., GAHLEITNER, M., BERNREITNER, H., "Mechanical and optical effects of elastomer interaction in polypropylene modification: ethylene-propylene rubber, poly-(ethylene-co-octene) and styrenebutadiene elastomers”, Express Polymer Letters, v. 6, n. 9, pp. 688-696, 2012.

[25] BOONSONG, K., SEADAN, M., LOPATTANANON., "Compatibilization of natural rubber (NR) and chloro sulfonated polyethylene (CSM) blends with zinc salts of sulfonated natural rubber”, Songklanakarin Journal Science Technol, Vol. 30, n.2, pp. 491-499, 2008. 
[26] SANGWICHIEN, C., SUMANATRAKOOL, P., PATARAPAIBOOLCHAI., "Effect of filler loading on curing characteristics and mechanical properties of thermoplastic vulcanizate”,Chiang Mai Journal of Science, vol.35, n.2, pp. 141-149, 2008.

[27] MIGUEL, J.J.P., Estudo comparativo das propriedades térmicas e mecânicas do polipropileno reforçado com óxido de zinco, carbonato de cálcio e talco, Dissertação de M.Sc., CCT/FIEB, Salvador, BA, Brasil, 2010.

[28] ARAÚJO, M. A. R., Avaliação de desempenho de artefato de poliestireno de alto impacto reciclado, Dissertação de M.Sc., CCT/UFRJ, Rio de Janeiro, RJ, Brasil, 2006.

[29] PEREIRA, N. C., BARBOSA, A. A., MILITÃO, R. A., INSTITUTO PRESBITERIANO MACKENZIE, http://www.mackenzie.br/7503.html, acessado abril de 2015.

[30] FERREIRA, L. A. S., PESSAN, L. A., JÚNIOR, E. H., “Comportamento mecânico e termo-mecânico de blendas poliméricas PBT/ABS”, Polímeros: Ciência e Tecnologia, v.19, n.3, pp. 67-72, 1997.

[31] QUENTAL, A. C., CARVALHO, F. P., TADA, E. S., et al., "Blendas de PHB e seus copolímeros: miscibilidade e compatibilidade”, Química Nova, v. 33, n. 2, pp. 438-446, 2010.

[32] MOREIRA, A. C. F., JÚNIOR, F O. C., SOARES, B. G.,"Morfologia co-contínua na mistura poliestireno/copolímero de etileno-acetato de vinila”, Polímeros: Ciência e Tecnologia, v. 12, n. 3, pp. 136146, 2002.

[33] GUO, Z., FANG, Z., TONG, L., “Application of percolation model on the brittle to ductile transition for polystyrene and polyolefin elastomer blends”, Express Polymer Letters, v. 1, n. 1, pp. 37-43, 2007.

[34] TANG, W., LIU, B., LIU, Z., et al., "Processing-dependent high impact polystyrene/styrene- butadienestyrene tri-block copolymer/carbon black antistatic composites”, Journal of Applied Polymer Science, v. 123, n.2 , pp. 1032-1039, 2012.

[35] RADONJIC, G., "Compatibilization effects of styrenic/rubber block copolymers in polypropylene/polystyrene blends”, Journal of Applied Polymer Science, v. 72, n.2, pp. 291-307, 1999.

[36] ZHANG, J., CHEN, H., ZHOU, Y., et al., "Compatibility of waste rubber powder/polystyrene blends by the addition of styrene grafted styrene butadiene rubber copolymer: effect on morphology and properties”, Polymer Bulletin, v. 10, n.10, pp. 2829-2841, 2013. 